\title{
Novel Measurement Technique for the Sagittal Vertical Axis and Its Clinical Application in Adult Spinal Deformity
}

\author{
Go Yoshida ${ }^{1}$, Kenta Kurosu ${ }^{1}$, Yu Yamato ${ }^{2}$, Tomohiko Hasegawa ${ }^{2}$, \\ Tatsuya Yasuda ${ }^{2}$, Daisuke Togawa ${ }^{2}$, Yukihiro Matsuyama $^{2}$ \\ ${ }^{1}$ Department of Orthopedic Surgery, Hamamatsu Medical Center, Hamamatsu, Japan \\ ${ }^{2}$ Department of Orthopedic Surgery, Hamamatsu Medical University, Hamamatsu, Japan
}

Study Design: Prospective physical measurement of the sagittal vertical axis (SVA).

Purpose: To evaluate a simple method for measuring SVA by analyzing its relationship with radiographic measurements and clinical appearance.

Overview of Literature: No studies have examined physical measurements using the cranial center of gravity (CCG) in a relaxed standing position.

Methods: The physical measurement of the horizontal distance between CCG and spina iliaca posterior superior (CCG-SIPS) was measured using a straight ruler in 252 healthy volunteers and 56 patients with adult spinal deformity. Health-related quality of life (HROOL) was evaluated using the Oswestry disability index (ODI), and clinical symptoms were assessed according to standing status and the presence of gastroesophageal reflux disease (GERD).

Results: CCG-SIPS increased with age in the volunteer group and strongly correlated with radiographic SVA in the patient group $(r=0.984)$. Differences increased between CCG-SIPS in patients in the relaxed position and radiographic SVA with an increase in sagittal malalignment $(r=0.692, p<0.001)$. ODI with high sagittal malalignment (CCG-SIPS $>120 \mathrm{~mm}$ ) was significantly larger in the patient group than in the group with low sagittal malalignment $(59.9 \pm 18.8$ vs. $45.1 \pm 17.0 ; p=0.004)$; these patients (CCG-SIPS $>120 \mathrm{~mm}$ ) needed crutches or walkers for standing. The patient group with GERD had significantly larger sagittal malalignment than the group without GERD (160.3 mm vs. $81.0 \mathrm{~mm}$ ).

Conclusions: The CCG-SIPS correlated with age and strongly reflected radiographic SVA and HROOL in the patients. Moreover, it reflects a relaxed posture without a backward shift in the radiographic position even in patients with severe sagittal malalignment. The critical limit of CCG-SIPS can be relevant to clinical appearance, including standing assistance $(>120 \mathrm{~mm})$ and the existence of GERD (>150 mm). Thus, it will be a useful predictor of true SVA in clinical practice before radiographic evaluation.

Keywords: Sagittal alignment; Sagittal vertical axis; Adult spinal deformity, Cranial center of gravity; Gastroesophageal reflux

\section{Introduction}

In the management of adult spinal deformity (ASD), as- sessment of spinal alignment is a time-tested tool and is generally performed using radiography with consequent radiation exposure. Two important concerns for radio-

\footnotetext{
Received Jun 7, 2016; Revised Jul 23, 2016; Accepted Aug 13, 2016

Corresponding author: Go Yoshida

Department of Orthopedic Surgery, Hamamatsu Medical Center,

328 Tomizuka-cho Naka-ku, Hamamatsu-city, Shizuoka 432-8580, Japan

Tel: +81-53-453-7111, Fax: +81-53-452-9217, E-mail: goy@K6.dion.ne.jp
} 
graphic measures are the patient's stance and evaluation range, which is the most reproducible and accurate position reflecting the patient's relaxed positon, and the extent to which the entire spine alignment should be evaluated. The former is proposed as the fists-on-clavicle position, which allows the visualization of the spinal and pelvic sagittal morphology without interference from the arms [1$3]$. However, this position may have a significant negative shift compared with a relaxed stance [4-6], and the degree of shift may increase when high sagittal imbalance exists. The latter refers to the use of the standardized C7 plumb line and its relationship with the posterior aspect of the S1 superior endplate. However, this omits the head position and craniocervical alignment. In true global sagittal balance, the head position should be considered in relation to the entire spine and pelvis in a relaxed standing position.

Recently, the sagittal vertical axis (SVA) and the cranial center of gravity (CCG) have been advocated as useful markers for evaluating global spine balance [7-9] and may be the most visible external landmarks in the lateral relaxed standing position. CCG was initially evaluated by Vital and Senegas as anatomical bases of isolated formolized heads by the suspension method; CCG is situated at the middle of the nasion-inion line, behind the sella turcica, above and slightly in front of the external auditory meatus [10]. The spina iliaca posterior superior (SIPS) is also visible and easily palpable. Thus, physically measuring the horizontal distance between CCG and SIPS (CCGSIPS) seems a optical method for measuring the true SVA in a relaxed standing position.

High sagittal malalignment not only influences healthrelated quality of life (HRQOL) of musculoskeletal disorders but can also cause standing disability or visceral disorders. Sagittal deformities have been principally associated with gastroesophageal reflux disease (GERD) [1113]. Furthermore, the prevalence of GERD is increasing in aging societies, and the interaction between ASD and GERD has become more important for orthopedic surgeons and physicians.

The aim of this study was to examine whether CCG-SIPS reflects sagittal alignment and to analyze its relationship with the existing radiographic measurements and clinical manifestations, including standing status and GERD.

\section{Materials and Methods}

After obtaining Institutional Review Board approval, a prospective analysis was performed in 252 healthy Japanese volunteers and 56 patients with ASD recruited from the Hamamatsu Medical Center. Healthy volunteers consisted of medical doctors, nurses, assistants from the hospital, and medical students without a history of spinal disorders. Patients with ASD were recruited from office consultation from May 2013 to May 2015. The inclusion criteria for patients with ASD were as follows: (1) age $>50$ years, (2) Cobb angle $>20^{\circ}$, (3) C7-SVA $>50 \mathrm{~mm}$, or (4) pelvic tilt $(\mathrm{PT})>25^{\circ}$, or $\mathrm{TK}>60^{\circ}$ and no previous history of spinal surgery.

Physical measurements using CCG-SIPS were measured in both a control group of normative subjects and a patient group with ASD. For both groups, measurements were obtained by hand using a precise straight ruler (Shinwa Rular, Shinwa Rules Co. Ltd., Niigata, Japan) with $90^{\circ}$ bent tips (error within $1 \mathrm{~mm}$ ) in a relaxed standing position with the arms extended and hands on either side (Fig. 1). The subject stood in parallel against the wall, and the trunk and pelvis were at right angles to avoid the different rotational positions. Although the relaxed position of patients with ASD can be affected by neck and head position, it was controlled by placing the neck in a comfortable resting position and having the patient face forward and look straight ahead with a horizontal gaze.

The CCG was approximated at the anterior margin of the external auditory canal. It was defined as a point approximately $1 \mathrm{~cm}$ above and anterior to the external auditory canal, which was nearly equal to the midpoints of the nasion-inion line (root of the nose to the outer cortex of the external occipital protuberance). The SIPS was carefully palpated by the examiner, and the CCG-SIPS was directly measured by two experienced spinal surgeons.

Radiological measurements were assessed only in the patient group. Standing radiographic measurements were obtained in three standard radiographic views: anteroposterior and lateral craniopelvic views from the head to pelvis, and a lateral pelvic view. These radiographic views were taken with a $1.5-\mathrm{m}$ distance between the $\mathrm{X}$-ray tube and the film for all patients. The standing position was strictly standardized and involved relaxing the head position with the subject looking straight ahead without pulling in the chin, and in the fist-on-clavicle position. After digitally obtained radiographs were transferred as the Digital Imaging and Communications in Medicine data to a computer, all parameters were measured by two examiners using an imaging software, and the results were aver- 

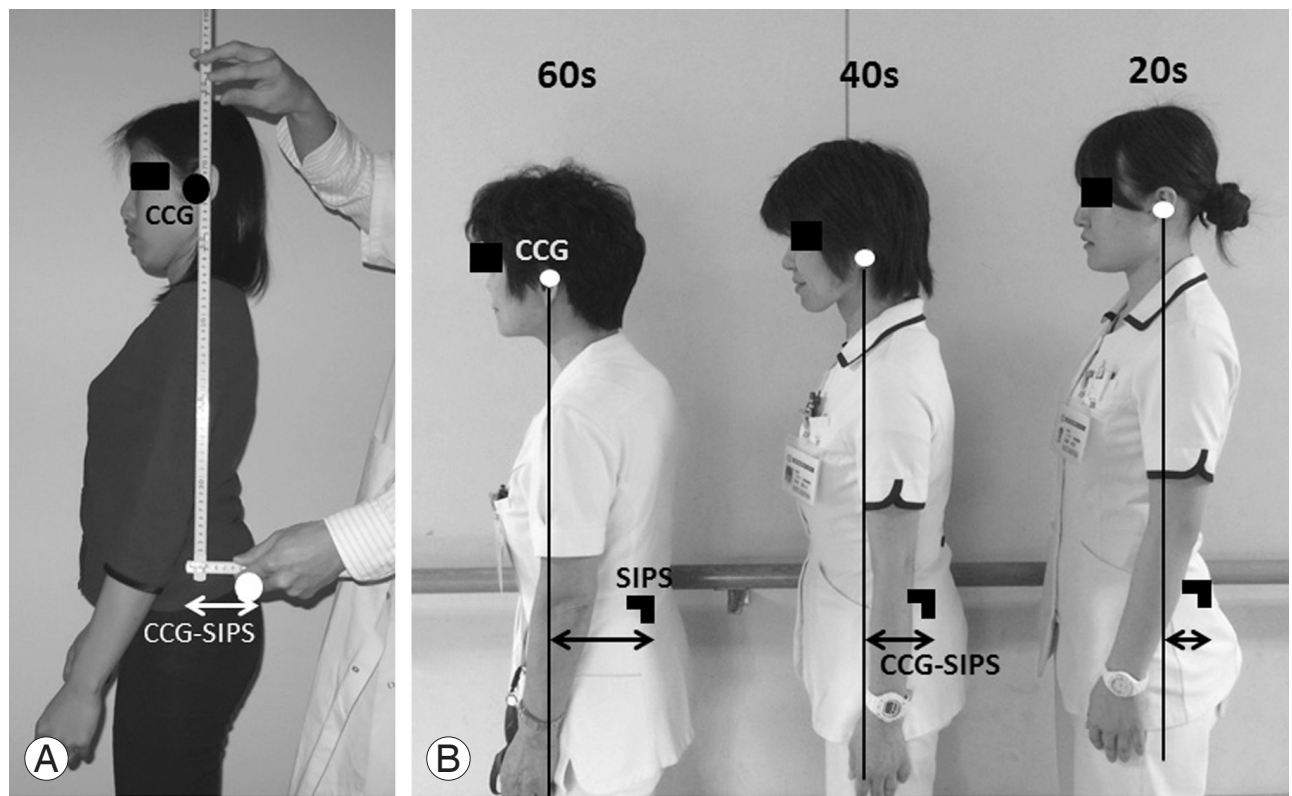

Fig. 1. The cranial center of gravity (CCG) was approximately located at the anterior margin of the external auditory canal (A). The spina iliaca posterior superior (SIPS) was carefully palpated by the examiner, and CCG-SIPS was directly measured with a precise ruler by the examiners. The horizontal distance between the CCG-SIPS in three different decades (B).

aged. Radiographs were only included if both the authors agreed that the radiographs were of an adequate quality to be used in this study.

The following spinal parameters were assessed: (1) CCG-C7SVA (distance between the CCG plumb line and the posterosuperior corner of C7); (2) C7-SVA (distance between the $\mathrm{C} 7$ plumb line and the posterosuperior aspect of S1); (3) CCG-SVA (distance between the CCG plumb line and the posterosuperior aspect of S1); (4) C2-C7 lordosis angle (Cobb angle between C2 and C7); (5) thoracic kyphosis (TK; Cobb angle between T4 and T12); (6) lumbar lordosis (LL; Cobb angle between T12 and S1); (7) PT (the angle between the vertical line and the line passing through the midpoint of the sacral plate to femoral heads axis); (8) pelvic incidence (PI; the angle between the line perpendicular to the sacral plate at its midpoint and the line connecting this point to the femoral head axis); and (9) sacral slope (SS; the angle between the horizontal and sacral plate).

The intraobserver and interobserver reliability were calculated using the reliability statistics by intraclass correlation (ICC) for the physical measurement of CCG-SIPS in the initial 100 control subjects, and the radiographic measurement of CCG-SVA in 56 patients in this study. The ICC values were graded using previously described semiquantitative criteria as follows: excellent for values in the range $0.9-1.0$, good for $0.7-0.89$, fair/moderate for $0.50-0.69$, low for $0.25-0.49$, and poor for 0.0-0.24.

The Oswestry disability index (ODI), a measure of locomotion, and the presence of GERD were used to evaluate HRQOL. Standing status was classified as follows: (A) standing without any kind of assistance or (B) standing with a crutch or a walker. Patients with GERD had already been diagnosed by a gastroenterologist using the frequency scale for symptoms of GERD (FSSG) questionnaire [14] (score of $\geq 8$ points) or upper endoscopy, and were being treated with oral medications. Subjects were divided into GERD positive (+) and GERD negative (-) groups.

SPSS ver. 14.0 (SPSS, Chicago, IL, USA) was used for statistical analyses. Student's $t$ test and Pearson's correlation coefficient test were used to examine differences in continuous variables between the two groups and correlations between the two parameters, respectively. Values are expressed as means with standard deviations with a statistical significance level set at $p<0.05$.

\section{Results}

\section{The reliability and reproducibility of physical mea- surements of CCG-SIPS}

The reliability statistics by ICC of CCG-SIPS in the initial 
100 control subjects for the intraobserver and interobserver reliability were 0.838 (95\% confidence interval [CI], $0.786-0.888$ ) and 0.787 (95\% CI, 0.683-0.857), respectively. ICCs of radiographic CCG-SVA in 56 patients for the intraobserver and interobserver reliability were 0.966 (95\% CI, 0.943-0.979) and 0.911 (95\% CI, 0.859-0.937), respectively. ICCs of CCG-SIPS were inferior compared with ICCs of radiographic SVA; however, these were good for the values previously described using the semiquantitative criteria.

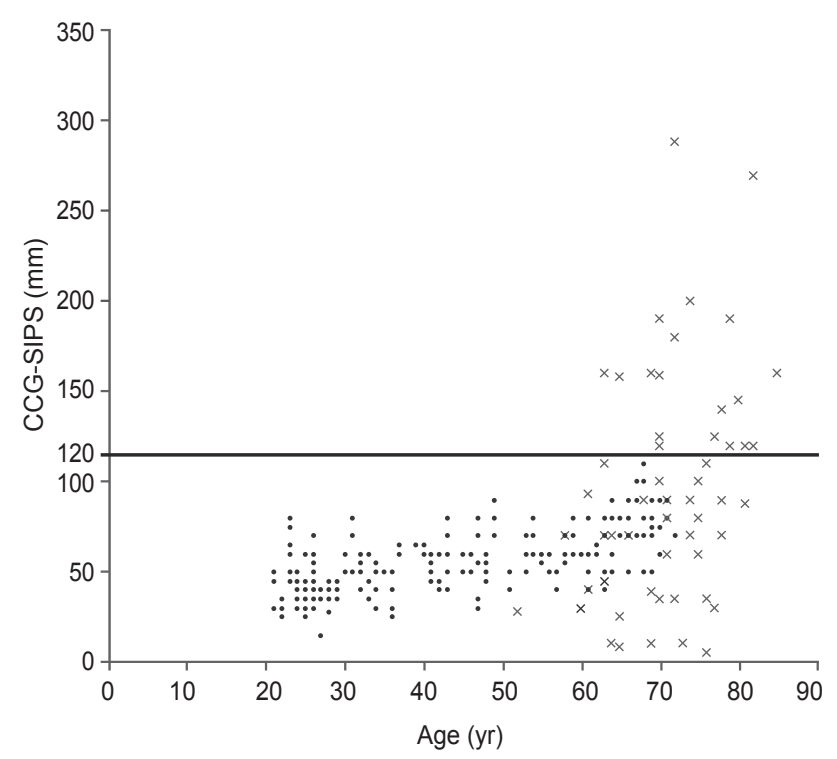

Fig. 2. Age-related correlation of the cranial center of gravity and spina iliaca posterior superior (CCG-SIPS) in 252 healthy volunteers and 56 patients with adult spinal deformity. The correlation coefficient (r) between age and CCG-SIPS was 0.5743 in the control and 0.380 in the patient group. We set the critical limit of CCG-SIPS as $120 \mathrm{~mm}$ because by using this critical limit, $100 \%$ of the controls in this study could be precisely divided.

\section{Age-related correlation of CCG-SIPS}

The mean CCG-SIPS in the control and patient groups were $54.3 \mathrm{~mm}$ (standard deviation [SD], $16.9 \mathrm{~mm}$; range, $15-110 \mathrm{~mm}$ ) and $93.8 \mathrm{~mm}(\mathrm{SD}, 63.7 \mathrm{~mm}$; range, 8-289 $\mathrm{mm})$, respectively. Age-related correlations were observed in both the control and patient groups. The correlation coefficient $(r)$ between age and CCG-SIPS was 0.574 $(p<0.001)$ in the control and $0.380(p=0.003)$ in the patient group (Fig. 2). The mean CCG-SIPS values in the control groups divided by patient age (three subgroups) are shown in Table 1 (age ranges, 21-39 [n=130], 40-59 $[n=71]$, and $\geq 60$ years $[n=51])$. There were no significant differences in the mean body mass index (BMI) among these subgroups; however, there were significant differences in the mean CCG-SIPS among the three groups $(p<0.05)$.

\section{Correlation between CCG-SIPS and radiographic spinopelvic parameters}

The CCG-SIPS was strongly correlated with CCGSVA and C7-SVA ( $r=0.984$, adjusted $R^{2}=0.961, p<0.001$; $r=0.967$, adjusted $R^{2}=0.925, p<0.001$, respectively) in ASD patients because the point of PSIS was close to the posterior aspect of the $\mathrm{S} 1$ superior endplate in lateral radiography. CCG-SIPS slightly correlated with CCG-C7SVA and LL ( $r=0.473$, adjusted $R^{2}=0.248, p<0.001$ and $r=-0.391$, adjusted $R^{2}=0.162, p=0.002$, respectively), but did not correlate with cervical lordosis, TK, SS, PT, and PI (Table 2 ). When the patient's stance between relaxed standing position and the fist-on-clavicle position were similar, theoretically, CCG-SIPS values were approximately equal

Table 1. CCG-SIPS in 252 asymptomatic volunteers

\begin{tabular}{|c|c|c|c|c|}
\hline \multirow{2}{*}{ Variable } & \multirow{2}{*}{ Total } & \multicolumn{3}{|c|}{ Age (yr) } \\
\hline & & $21-39$ & $40-59$ & $>60$ \\
\hline No. of cases & 252 & 130 & 71 & 51 \\
\hline Age (yr) & $41.1 \pm 16.2$ & $27.3 \pm 4.5$ & $48.6 \pm 6.2$ & $65.9 \pm 3.3$ \\
\hline Sex (male/female) & $111 / 141$ & $44 / 86$ & $35 / 36$ & $32 / 19$ \\
\hline Body mass index $\left(\mathrm{kg} / \mathrm{m}^{2}\right)$ & $21.8 \pm 2.4$ & $20.8 \pm 2.2$ & $22.0 \pm 1.8$ & $23.8 \pm 2.3$ \\
\hline CCG-SIPS (mm) & $54.3 \pm 17.2$ & $46.3 \pm 14.3$ & $57.4 \pm 12.7^{\text {a) }}$ & $70.7 \pm 15.6^{b}$ \\
\hline
\end{tabular}

Values are presented as mean \pm standard deviation

CCG-SIPS, horizontal distance between cranial center of gravity and spina iliac posterior superior.

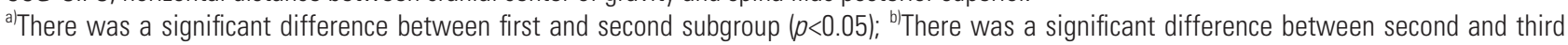
subgroup $(p<0.05)$. 
Table 2. Correlation between CCG-SIPS and radiographic parameters in ASD patients

\begin{tabular}{lccc} 
Parameter & $r$ & Adjusted $R^{2}$ & $p$-value \\
CCG-SIPS vs. CCG-SVA & 0.984 & 0.961 & $<0.001$ \\
CCG-SIPS vs. C7-SVA & 0.967 & 0.925 & $<0.001$ \\
\hline CCG-SIPS vs. CCG-C7SVA & 0.473 & 0.248 & $<.001$ \\
CCG-SIPS vs. CL & 0.181 & 0.018 & NS \\
\hline CCG-SIPS vs. TK & -0.217 & 0.030 & NS \\
CCG-SIPS vs. LL & -0.391 & 0.162 & 0.002 \\
CCG-SIPS vs. SS & -0.053 & - & NS \\
CCG-SIPS vs. PT & 0.036 & - & NS \\
\hline CCG-SIPS vs. PI & -0.060 & - & NS \\
\hline
\end{tabular}

CCG-SIPS, horizontal distance between cranial center of gravity (CCG) and spina iliac posterior superior; ASD, adult spinal deformity; CCG-SVA, distance between the CCG plumb line and the posterosuperior aspect of S1; C7-SVA, distance between the C7 plumb line and the posterosuperior aspect of S1; CCG-C7SVA, distance between the CCG plumb line and the posterosuperior corner of C7; CL, cervical lordosis; NS, not significant; TK, thoracic kyphosis; LL, lumbar lordosis; SS, sacral slope; PT, pelvic tilt; PI, pelvic incidence.

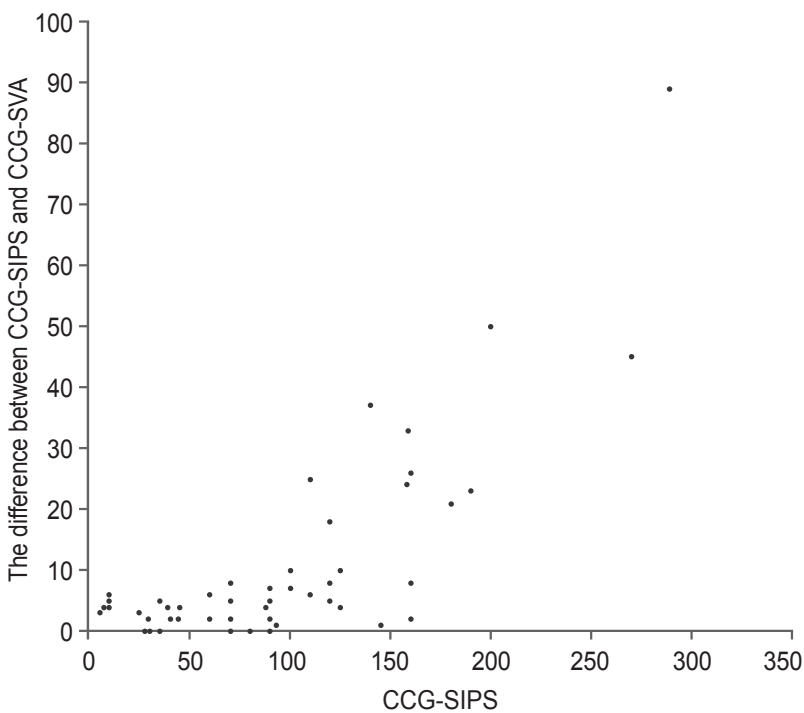

Fig. 3. Differences between the cranial center of gravity and spina iliaca posterior superior (CCG-SIPS) and CCG-sagittal vertical axis (SVA). The difference increased (same as CCG-SIPS and CCG-SVA) with increase in CCG-SIPS $(r=0.692, p<0.001)$.

to CCG-SVA based on radiographic measurements. However, differences increased between CCG-SIPS and CCG-SVA (same as CCG-SIPS minus CCG-SVA) with an increase in CCG-SIPS $(r=0.692, p<0.001)$ (Figs. 3,4$)$.

\section{Correlation between CCG-SIPS and HRQOL}

CCG-SIPS was not significantly correlated with ODI in all 56 patients with ASD $(r=0.154, p=0.256)$. We set the critical limit of CCG-SIPS as $120 \mathrm{~mm}$ because in using this
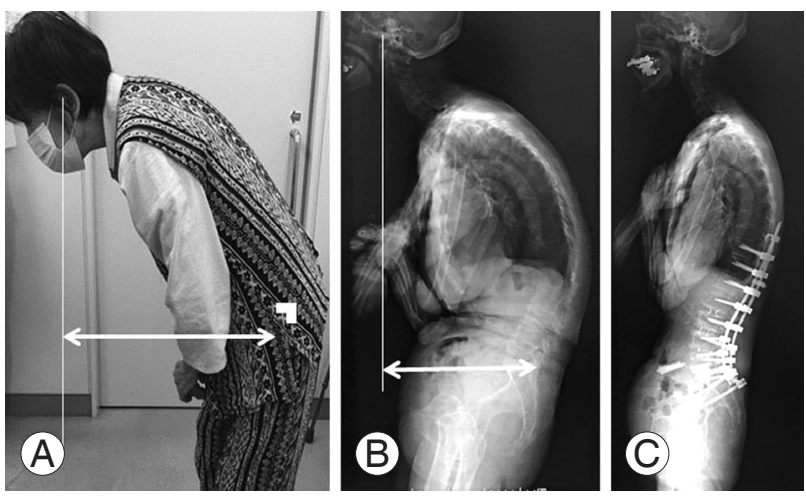

Fig. 4. Radiographs of a 72-year-old female patient who underwent corrective spinal surgery following adult spinal deformity. She underwent L2-L5 oblique lateral interbody fusion and posterior T10-iliac fusion with L5-S1 posterior lumbar interbody fusion. Preoperative lateral photograph in a relaxed position demonstrating the inability to maintain a horizontal gaze due to severe kyphosis and severe sagittal malalignment (horizontal distance between cranial center of gravity and spina iliac posterior superior [CCG-SIPS] $=289 \mathrm{~mm}$ ) (A). Preoperative lateral radiograph of the entire spine showing a negative shift compared to relaxed stance (CCG-sagittal vertical axis [SVA]=200 mm) (B). Postoperative lateral radiograph obtained at 1-year follow-up (CCG-SIPS=CCG-SVA=55 mm) (C).

critical limit, $100 \%$ of the controls in this study could be strictly divided. Therefore, we grouped patients according to CCG-SIPS as follows: $<120 \mathrm{~mm}$ (low to medial sagittal imbalance, $\mathrm{n}=37$ ) or $\geq 120 \mathrm{~mm}$ (high sagittal imbalance, $\mathrm{n}=19$ ). Patients with high sagittal imbalance had significantly larger ODI than those with low sagittal imbalance (ODI; $59.9 \pm 18.8$ and $45.1 \pm 17.0$, respectively; $p=0.004$ ). 
Table 3. Clinical appearance of CCG-SIPS for standing or GERD

\begin{tabular}{|c|c|c|c|c|c|c|}
\hline \multirow{2}{*}{ Variable } & \multicolumn{3}{|c|}{ Standing status } & \multicolumn{3}{|c|}{ GERD } \\
\hline & Group A & Group B & $p$-value & GERD- & GERD+ & $p$-value \\
\hline No. of cases & 29 & 27 & & 47 & 9 & \\
\hline Age (yr) & $67.7 \pm 6.9$ & $74.1 \pm 5.8$ & $<0.001$ & $70.1 \pm 7.4$ & $74.3 \pm 3.5$ & 0.103 \\
\hline CCG-SIPS (mm) & $51.2 \pm 29.7$ & $139.5 \pm 58.6$ & $<0.001$ & $81.0 \pm 55.6$ & $160.3 \pm 64.8$ & $<0.001$ \\
\hline CCG-SVA (mm) & $48.0 \pm 29.2$ & $122.0 \pm 46.3$ & $<0.001$ & $74.7 \pm 51.2$ & $132.7 \pm 42.4$ & 0.002 \\
\hline $\operatorname{LL}\left({ }^{\circ}\right)$ & $32.6 \pm 14.2$ & $21.6 \pm 12.2$ & 0.002 & $29.7 \pm 13.3$ & $11.8 \pm 6.0$ & $<0.001$ \\
\hline
\end{tabular}

CCG-SIPS, horizontal distance between cranial center of gravity (CCG) and spina iliac posterior superior; GERD, gastroesophageal reflux disease; SVA, sagittal vertical axis; LL, lumbar lordosis.

\section{Clinical appearance of CCG-SIPS for standing or GERD}

The results of standing status including (A) standing without any kind of assistance and (B) standing with crutch or walker were 29 and 27, respectively. The mean CCGSIPS in groups A and B were $51.2 \mathrm{~mm}$ and $139.5 \mathrm{~mm}$, respectively. There were significant differences in the mean CCG-SIPS, CCG-SVA, and LL of groups A and B $(p<0.05)$. We also set the critical limit of standing assistance, that is, the ideal cut-off value of CCG-SIPS between groups A and $B$ at $120 \mathrm{~mm}$. Using this critical limit, $100 \%$ of group A and $74 \%$ of group B could be divided precisely. The number of GERD+ and GERD- patients were 9 and 47, respectively. The mean CCG-SIPS in GERD+ and GERDwere $160.3 \mathrm{~mm}$ and $81.0 \mathrm{~mm}$, respectively. There were significant differences in the mean CCG-SIPS, CCG-SVA, and LL between GERD+ and GERD- $(p<0.05)$. We set the critical limit of GERD, which is the ideal cut-off value of CCG-SIPS with or without GERD, at $150 \mathrm{~mm}$. Using this critical limit, $66.7 \%$ of GERD+ and $87.2 \%$ of GERD-patients could be divided precisely (Table 3 ).

\section{Discussion}

A number of studies have sought to evaluate the effect of patient stance and arm position on SVA, to identify an optimal, functional position. Results of these studies indicate that some positions may be better than others in terms of variance in SVA readings; however, none of the positions represented a functional standing position [1-6]. Although radiographic measurement using the plumb line offset is simple, it may not accurately reflect the relaxed, standing position of the spine. Considering the risks asso- ciated with radiation exposure from repeated radiographs, there is an increasing need for a more objective and less invasive assessment to monitor spinal alignment. Other methods for measuring spinal balance have been also developed, such as the photogrammetry, gravity line using a force plate, and surface tracking systems [15-18]. However, these methods are not obtained from optical assessments, could not undergo quantitative evaluation for head position and cervical alignment, are time consuming, and could only be applied in laboratory environments. In this study, we measured SVA using CCG-SIPS as an optical method and less invasive fashion, and revealed age-related correlations with CCG-SIPS in healthy volunteers. The normative value of CCG-SIPS is $54.3 \mathrm{~mm}$ and CCG may have been measured near the hip axis or S1 posterosuperior endplate in cases where there is no sagittal imbalance. However, in patients with high sagittal imbalance, significant differences between CCG-SIPS and CCG-SVA were observed (Fig. 3). Previous studies also referred to this backward shift in radiographic standing position of patients compared to those in a relaxed position [4-6]. Therefore, surgeons should consider the true image of the patient's relaxed standing position aside from radiographic values in the presence of severe sagittal malalignment.

Severe sagittal malalignment influences HRQOL, although this study could not indicate the linear correlation between the CCG-SIPS and ODI. However, the ODI with high sagittal malalignment (CCG-SIPS >120 mm) was significantly larger than that with low sagittal malalignment. Severe sagittal malalignment influences HRQOL because it may lead to falls as well as visceral disorders. Falling in elderly patients is a major cause of death, fractures, and traumatic brain injuries that affect quality of life and independent living. The mechanisms by which indi- 
viduals develop sagittal imbalance vary and will depend on specific compensatory mechanisms from CCG to the toe. The body adapts to a change in balance to regulate the center of gravity over the narrowest perimeter possible. Thus, ASD patients have to compensate by pelvic retroversion to reposition their head over the femoral head and maintain a horizontal gaze [9]. However, in this study, individuals with severe sagittal malalignment (estimated by CCG-SIPS or CCG-SVA >120 mm) could stand alone and required walking assistance.

The prevalence of ASD was suggested to be up to $60 \%$ among elderly individuals [19]. Additionally, recent reports have considered this condition to be as life-threatening as other important chronic diseases [20]. Therefore, not only spinal surgeons but also general physicians may have to deal with ASD during their first contact with the patient. Sagittal deformities have been associated with GERD [11,12]. After evaluating GERD in patients with osteoporosis by using FSSG with radiological parameters from total spine lateral radiographs, it was found that the angle of lumbar kyphosis and the number of lumbar vertebral fractures were significantly associated with GERD. Especially, the lumbar lordosis angle, sagittal alignment and strength of the back muscles were reported to affect the presence of GERD symptoms as measured by FSSG [13]. GERD is a chronic disorder, involving the retrograde flow of the gastro-duodenal contents into the esophagus, causing heartburn and acid regurgitation. The prevalence of GERD is increasing in these aging societies. However, the findings of previous studies may not have been broadly applicable. In this study, ASD patients with concomitant GERD symptoms (16.1\% of total ASD patients) had significantly larger sagittal malalignment than those without GERD symptoms. Additionally, we also found that high sagittal malalignment (CCG-SIPS $>150 \mathrm{~mm}$ ) is an important predictor related to the presence of GERD. Furthermore, we found that this knowledge was also useful for the better understanding of general physicians.

This study had several limitations. First, it is possible that some measurements were subject to error. For example, fatty tissue may influence the measurements in cases of high BMI. In this study, the control group had relatively low BMI, although there was no significant difference between the groups in terms of BMI. Second, there might be an important lack of the impact of the body rotation, which influences the measurement of the distance between CCG and SIPS. Third, not all items for assess- ing the spinal sagittal curvature and PT were evaluated. However, limiting measurements to SVA, we consider that our results should be useful to understand global spinal balance without the need for radiation exposure. Finally, clinical assessments using the CCG-SIPS, including preoperative and postoperative assessments after corrective spinal surgery for ASD, were not performed because of short follow-up periods and low number of the patients. Moreover, this study included only a cross-sectional optical assessment and did not evaluate dynamic changes in spinal alignment. Therefore, motion or gait analysis of spinal alignment by age as a longitudinal design should be investigated.

\section{Conclusions}

In conclusion, the CCG-SIPS was a reliable value that correlated with the radiographic measurement of SVA. This finding may be useful not only for spine surgeons but also for general physicians, and it is a potential index marker of sagittal alignment without the need for radiation exposure.

\section{Conflict of Interest}

No potential conflict of interest relevant to this article was reported.

\section{References}

1. Marks MC, Stanford CF, Mahar AT, Newton PO. Standing lateral radiographic positioning does not represent customary standing balance. Spine (Phila Pa 1976) 2003;28:1176-82.

2. Faro FD, Marks MC, Pawelek J, Newton PO. Evaluation of a functional position for lateral radiograph acquisition in adolescent idiopathic scoliosis. Spine (Phila Pa 1976) 2004;29:2284-9.

3. Horton WC, Brown CW, Bridwell KH, Glassman SD, Suk SI, Cha CW. Is there an optimal patient stance for obtaining a lateral 36 " radiograph? A critical comparison of three techniques. Spine (Phila $\mathrm{Pa}$ 1976) 2005;30:427-33.

4. Zheng X, Chaudhari R, Wu C, Mehbod AA, Transfeldt EE, Winter RB. Repeatability test of C7 plumb line and gravity line on asymptomatic volunteers using an optical measurement technique. Spine (Phila 
Pa 1976) 2010;35:E889-94.

5. Aota Y, Saito T, Uesugi M, Ishida K, Shinoda K, Mizuma K. Does the fists-on-clavicles position represent a functional standing position? Spine (Phila Pa 1976) 2009;34:808-12.

6. Park SM, Song KS, Park SH, Kang H, Daniel Riew $\mathrm{K}$. Does whole-spine lateral radiograph with clavicle positioning reflect the correct cervical sagittal alignment? Eur Spine J 2015;24:57-62.

7. Tang JA, Scheer JK, Smith JS, et al. The impact of standing regional cervical sagittal alignment on outcomes in posterior cervical fusion surgery. Neurosurgery 2012;71:662-9.

8. Sugrue PA, McClendon J Jr, Smith TR, et al. Redefining global spinal balance: normative values of cranial center of mass from a prospective cohort of asymptomatic individuals. Spine (Phila $\mathrm{Pa} 1976$ ) 2013;38:484-9.

9. Yoshida G, Yasuda T, Togawa D, et al. Craniopelvic alignment in elderly asymptomatic individuals: analysis of 671 cranial centres of gravity. Spine (Phila Pa 1976) 2014;39:1121-7.

10. Vital JM, Senegas J. Anatomical bases of the study of the constraints to which the cervical spine is subject in the sagittal plane: a study of the center of gravity of the head. Surg Radiol Anat 1986;8:169-73.

11. Miyakoshi N, Kasukawa Y, Sasaki H, Kamo K, Shimada Y. Impact of spinal kyphosis on gastroesophageal reflux disease symptoms in patients with osteoporosis. Osteoporos Int 2009;20:1193-8.

12. Yamaguchi T, Sugimoto T, Yamada H, et al. The presence and severity of vertebral fractures is associated with the presence of esophageal hiatal hernia in post- menopausal women. Osteoporos Int 2002;13:331-6.

13. Imagama S, Hasegawa $Y$, Wakao N, Hirano K, Hamajima N, Ishiguro N. Influence of lumbar kyphosis and back muscle strength on the symptoms of gastroesophageal reflux disease in middle-aged and elderly people. Eur Spine J 2012;21:2149-57.

14. Kusano M, Shimoyama Y, Sugimoto S, et al. Development and evaluation of FSSG: frequency scale for the symptoms of GERD. J Gastroenterol 2004;39:888-91.

15. Roussouly P, Gollogly S, Noseda O, Berthonnaud E, Dimnet J. The vertical projection of the sum of the ground reactive forces of a standing patient is not the same as the $\mathrm{C} 7$ plumb line: a radiographic study of the sagittal alignment of 153 asymptomatic volunteers. Spine (Phila Pa 1976) 2006;31:E320-5.

16. Schwab F, Lafage V, Boyce R, Skalli W, Farcy JP. Gravity line analysis in adult volunteers: age-related correlation with spinal parameters, pelvic parameters, and foot position. Spine (Phila Pa 1976) 2006;31:E959-67.

17. Pazos V, Cheriet F, Danserau J, Ronsky J, Zernicke RF, Labelle H. Reliability of trunk shape measurements based on 3-D surface reconstructions. Eur Spine J 2007;16:1882-91.

18. Wong WY, Wong MS. Trunk posture monitoring with inertial sensors. Eur Spine J 2008;17:743-53.

19. Schwab F, Dubey A, Gamez L, et al. Adult scoliosis: prevalence, SF-36, and nutritional parameters in an elderly volunteer population. Spine (Phila Pa 1976) 2005;30:1082-5.

20. Pellise F, Vila-Casademunt A, Ferrer M, et al. Impact on health related quality of life of adult spinal deformity (ASD) compared with other chronic conditions. Eur Spine J 2015;24:3-11. 